\title{
Reappearance of structure in colloidal suspensions
}

\author{
L. F. Rojas ${ }^{1}$, C. Urban ${ }^{1,2}$, P. Schurtenberger ${ }^{1}$, \\ T. GISLER ${ }^{3}$ and H. H. VON GRÜNBERG ${ }^{3}$ \\ 1 Université de Fribourg, Institut de Physique - Pérolles, CH-1700 Fribourg, Switzerland \\ 2 LS Instruments - Chemin du Musée 3, CH-1700 Fribourg, Switzerland \\ 3 Universität Konstanz, Fachbereich Physik - D-78457 Konstanz, Germany
}

(received 25 March 2002; accepted in final form 23 September 2002)

PACS. 82.70.Dd - Colloids.

PACS. 61.20.Qg - Structure of associated liquids: electrolytes, molten salts, etc.

\begin{abstract}
Static structure factors $S(q)$ of deionized aqueous suspensions of charged polystyrene particles with similar radii but strongly differing bare charges have been measured for volume fractions $3.5 \times 10^{-4} \leq \varphi \leq 1.55 \times 10^{-2}$, using a cross-correlation light scattering technique which allows for the extraction of single scattered light from highly turbid samples. Measurement of absolute intensities allowed to determine unambiguously normalized values of $S(q)$. With decreasing volume fraction, the amplitude of the first peak of $S(q)$ reflecting the order of the suspension does not monotonically decrease, but rather shows a distinct minimum at $\varphi \sim(5-8) \times 10^{-3}$. This reappearance of structure is compared with theoretical predictions on the basis of a jellium model for the effective interparticle potential $U_{\text {eff }}(r)$.
\end{abstract}

The forces governing the structure and phase behavior of suspensions of charged colloidal particles are of prime importance for many technological applications, such as ceramic processing, drug delivery or the stability control of paints. In the standard picture of Derjaguin, Landau, Verwey, and Overbeek (DLVO) [1], the electrostatic interaction between two charged colloidal particles in an electrolyte arises from the repulsion of the electric double layers that form due to the competition between configurational entropy of the small ions and their potential energy in the Coulomb field of the macroions with negative charge $-Z e_{0}, e_{0}$ being the elementary charge. When the bare charge $Z$ is large, a part of the surrounding counterions is strongly bound to the particle and thus reduces the bare charge to an effective charge $\tilde{Z}<Z$ which then governs the ion distribution at distances comparable to the Debye screening length $\kappa^{-1}[2]$. In contrast to the situation in simple liquids, however, the strength of the effective interparticle potential $U_{\text {eff }}(r)$ determined by the effective charge $\tilde{Z}$ and its range $\kappa^{-1}$ does not only depend on the bare charge $Z$ but also on the concentrations of co- and counterions and the particle volume fraction $\varphi[3]$. This density dependence of the effective potential is reflected by the fact that even at volume fractions as low as $10^{-4}$ strongly charged particles show considerable order, as reflected by the strong first peak in the colloid-colloid structure factor $S(q)$ measured in light scattering experiments from deionized suspensions [4,5]. When, at higher volume fractions, the number of counterions balancing the particle charge becomes larger than the number of excess salt ions, the screening will become dominated by the former, 
and increasing the macroion number density $n_{\mathrm{p}}$ will result in enhanced screening. This has a twofold effect: it will reduce $\kappa^{-1}$ and thus the range of the pair potential, but it will also affect a prefactor in $U_{\text {eff }}(r)$. Both effects together can cause an initial increase followed by a reduction of order as $\varphi$ increases. At still higher volume fractions, though, the packing of the macroions will finally be expected to dominate the structure of the suspension. The intrinsic density dependence of the interaction potential between charged colloidal particles can thus show a rather complex behavior, as the present investigation confirms. Starting from a dense suspension and measuring $S(q)$, we find that with decreasing $\varphi$ structure is first reduced, but reappears at lower volume fractions, in striking contrast to the situation in simple liquids where a reduction of the packing fraction always leads to a monotonic decrease of inter-particle structure. Although $S(q)$ has been measured for deionized bulk suspensions of well-characterized, highly charged particles using light, X-ray and neutron scattering [6], an investigation of the range of volume fractions where such a reappearance of structure might occur has to date been hampered, in particular for light scattering experiments, by the large scattering contrasts leading to strong multiple scattering when the experiments are performed with aqueous solvents.

In this paper we present an investigation of the structure of deionized suspensions of highly charged polystyrene particles. Static structure factors $S(q)$ have been measured over a wide range of volume fractions $3.5 \times 10^{-4} \leq \varphi \leq 1.55 \times 10^{-2}$ by light scattering combined with a cross-correlation scheme for the detection of single scattered light from the highly turbid samples. Absolute measurements of the single scattering intensities allow an unambiguous normalization of $S(q)$, even for the highest volume fractions, where $S(q)$ shows strong oscillations at the largest values of $q$ accessible with the light scattering experiment. Quantifying the order in the suspensions by the height of the first peak $S\left(q_{\max }\right)$ of the structure factor, we find comparable, non-monotonic $\varphi$-dependencies of $S\left(q_{\max }\right)$ for particles differing only in their bare charge $Z$.

For our experiments we used sulfate polystyrene latex particles (Interfacial Dynamics) with radii $a=54.9 \mathrm{~nm}$ and $a=58.7 \mathrm{~nm}$, respectively, whose charge is independent of $p \mathrm{H}$ and volume fraction. Accurate values of the particle size and of the size polydispersity were obtained from small-angle neutron scattering experiments; they agreed well with the values measured with static and dynamic light scattering from dilute samples and transmission electron microscopy. The samples were prepared by diluting the aqueous stock suspension into a mixture of ethanol and water containing a volume fraction of $70-75 \%$ pure ethanol. This reduces the solvent dielectric constant $\epsilon$ to values between 37.9 and 40.6 [7], resulting in a reduction of the screening length which is sufficient to prevent crystallization. Large aggregates and dust particles were removed by filtering the samples through $0.8 \mu \mathrm{m}$ cellulose filters into quartz glass cuvettes containing mixed bed ion exchange resin (BioRad). The cell caps were then sealed with Teflon tape and silicone putty; the degree of deionization was repeatedly checked by measuring the static light scattering at angles where a peak in the static structure factor was expected. Deionization was typically completed within 2 weeks.

The resulting samples were highly turbid, with turbidities between $0.5 \mathrm{~cm}^{-1}$ and $15.5 \mathrm{~cm}^{-1}$. In order to isolate single scattered light containing the direct information on $S(q)$ from the background arising from multiply scattered photons we employed a 3D cross-correlation scheme [8-11]; light scattered at angles $10^{\circ} \leq \vartheta \leq 150^{\circ}$ was collected by two single-mode fibers and detected by two silicon avalanche photodiodes (EG\&G) whose TTL output signals were cross-correlated by a multi-tau correlator (ALV). Details of the experimental setup are given in [12]. Single-scattering count rates $I(q)$ were determined from the reduction of the measured amplitude of the cross-correlation function of the raw count rates. A turbidity correction has been applied in order to account for the angle-dependent optical path length in the square sample cells. 

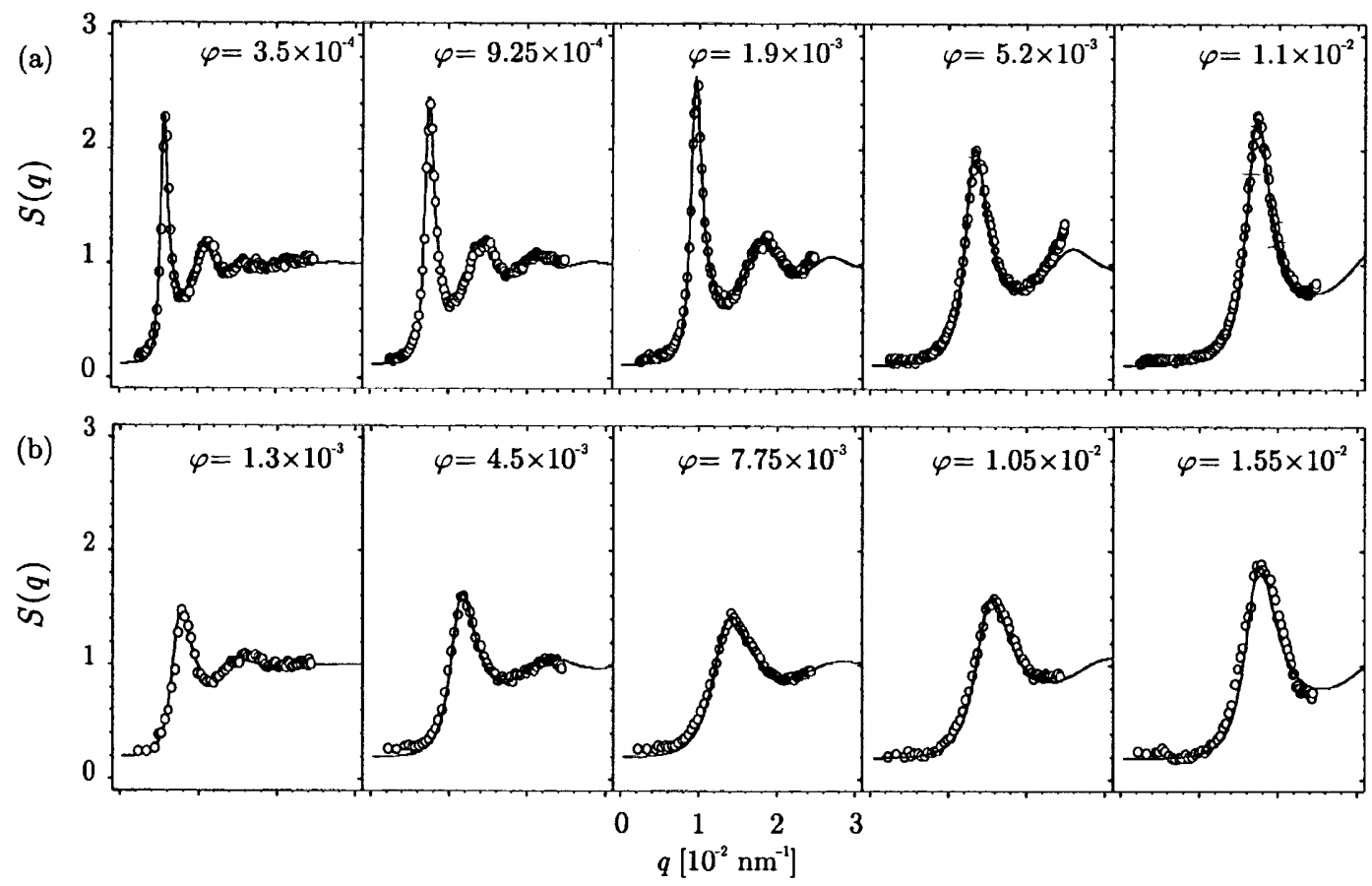

Fig. 1 - Static structure factors $S(q)$ of deionized suspensions of polystyrene latex particles measured with light scattering as a function of the magnitude of the scattering wave vector $q$ at $25^{\circ} \mathrm{C}$. (a) Average particle radius $a=54.9 \mathrm{~nm}$, size polydispersity $p=11.8 \%$, Bjerrum length $l_{\mathrm{B}}=1.383 \mathrm{~nm}$, and solvent refractive index $n=1.3645$. (b) $a=58.7 \mathrm{~nm}, p=15.7 \%, l_{\mathrm{B}}=1.482 \mathrm{~nm}, n=1.3656$. Particle volume fractions increase from left to right. Laser wavelengths were $\lambda_{0}=632.8 \mathrm{~nm}$ in (a) and $\lambda_{0}=680.4 \mathrm{~nm}$ in (b). Solid lines: best-fit polydisperse Rogers-Young calculations using a Yukawa interaction potential with adjustable volume fraction and effective charge.

With incident laser powers of $30-50 \mathrm{~mW}$ it typically took about $200-600$ s to get $I(q)$ to within $10 \%$ at a particular wave vector $q$. By normalization of the single-scattering count rate with the measured form factor $P(q)$ and the count rate of a dilute reference sample containing small polystyrene particles $(a=10.5 \mathrm{~nm})$ in the identical solvent, the quantity $c S(q)$ was computed; here, $c$ is the particle weight fraction. By fitting the peak position of a polydisperse Rogers-Young (RY) $S(q)[13]$ to the observed peak position of $c S(q)$ and using a particle density $\rho=1.05 \mathrm{~g} / \mathrm{cm}^{3}$, we determined the particle volume fraction $\varphi=c / \rho$, which in turn allowed to accurately determine $S(q)$; the differences between the resulting values of $\varphi$ and the values calculated from the volume fraction of the stock and the dilution factors are consistent with adsorption of a particle monolayer onto the ion exchange resin. Measured structure factors are shown in fig. 1.

For both particle sizes and bare charges the measured $S(q)$ show distinct liquid-like structure, with a strong first peak reflecting the well-defined interparticle separation; at the lowest volume fractions investigated, our data also show well-developed second- and third-order peaks with reduced heights. Interestingly, at low $q$ the structure factor decreases to values that compare very well to the RY calculations. This is remarkable insofar that at these low angles the contribution of single scattered photons to the total signal detected is as small as $20 \%$. Over a comparable range of volume fractions $1.3 \times 10^{-3} \leq \varphi \leq 1.55 \times 10^{-2}$ the structure factors of the 


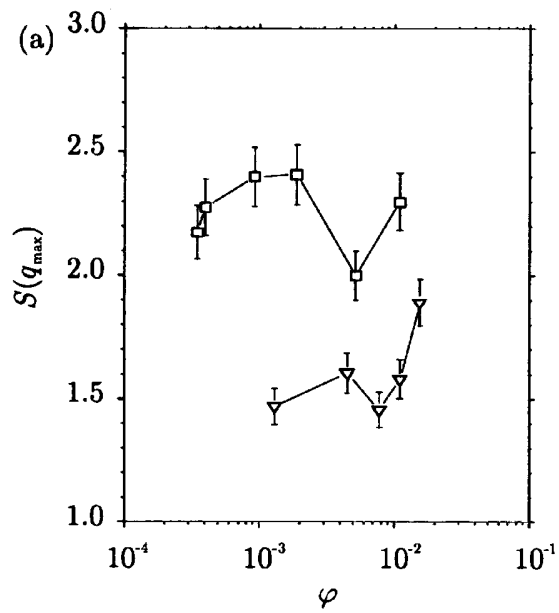

(b)

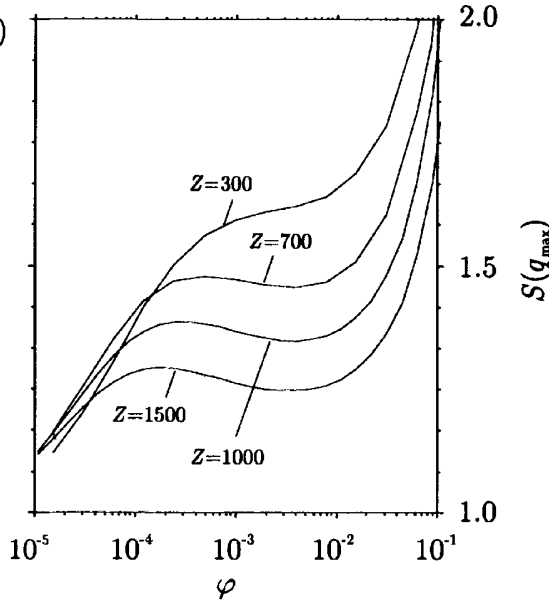

Fig. 2 - (a) Peak heights $S\left(q_{\max }\right)$ of the measured structure factors as a function of volume fraction $\varphi$ for particles with diameter $a=54.9 \mathrm{~nm}$ (squares) and $a=58.7 \mathrm{~nm}$ (triangles). Error bars represent standard deviations of $S\left(q_{\max }\right)$ due to photon statistics. (b) The height of the first peak of the colloid structure factor, $S\left(q_{\max }\right)$, of a charge-stabilized colloidal suspension as a function of the volume fraction $\varphi$ calculated solving the Ornstein-Zernike equation with the RMSA closure relation with effective pair potentials based on the jellium approximation. The curves are labelled with the respective bare colloidal charges $Z$.

larger particles with $a=58.7 \mathrm{~nm}$ show a similar behavior to that of the smaller ones, albeit with a less pronounced structure in $S(q)$; the larger values of $S(0)$ between 0.2 and 0.3 reflect not only the weaker coupling due to a smaller effective charge, but also the larger incoherent scattering due to the larger-size polydispersity. The rise of the peak height $S\left(q_{\max }\right)$ as $\varphi$ increases is in good agreement with earlier light scattering results by Wagner et al. [14] on smaller particles. At higher volume fractions between about $0.3 \%$ and $0.5 \%$, however, the structure factor peak drops significantly to what appears to be a minimum (see fig. 2a) at $\varphi \sim 5 \times 10^{-3}$ for the $a=54.9 \mathrm{~nm}$ particles and $\varphi \sim 8 \times 10^{-3}$ for the $a=58.7 \mathrm{~nm}$ particles; at higher volume fractions, $S\left(q_{\max }\right)$ is observed to increase again. Although both the Bjerrum lengths $l_{\mathrm{B}}$ and the particle sizes $a$ for the two data sets differ only by about $7 \%$, the amplitude of the $S\left(q_{\max }\right)$ data for the $a=58.7 \mathrm{~nm}$ particles is significantly smaller than the one of the slightly smaller particles; this indicates that their respective bare charges $Z$ do indeed grossly differ.

Such a non-monotonic dependence of $S\left(q_{\max }\right)$ on volume fraction has been predicted by the jellium model (JM) of Beresford-Smith et al. [3]. The density dependence of the structure factor is due to a density-dependent effective colloid-colloid interaction potential $U_{\text {eff }}(r)$. For an isolated pair of colloidal particles and at large distances $r$, this pair potential is known to be a Yukawa potential $\sim \bar{y}^{2} e^{-\kappa r} / r$ with a screening constant $\kappa$ and a prefactor $\bar{y}^{2}$ which is the square of the amplitude of the electrostatic mean-field potential around a single particle. For two colloidal particles in a suspension of finite concentration, the JM assumes the effective pair potential to be still Yukawa-like; however, both $\bar{y}$ and $\kappa$ now depend on the colloid density, leading ultimately to the $\varphi$-dependence of $S\left(q_{\max }\right)$. In the following, we briefly recall how to calculate $\bar{y}$ and $\kappa$ in the JM; for a complete derivation of the theory and a thorough discussion of the underlying assumptions we refer the reader to the original work of Beresford-Smith et al. [3].

The dependence of $\bar{y}$ and $\kappa$ on the colloid density $n_{\mathrm{p}}=3 \varphi / 4 \pi a^{3}$ originates from the fact that the total number of counterions in the system is proportional to the number of colloids. To 
determine $\bar{y}$, one first has to calculate the electrostatic potential $\Psi(r)$ (normalized by $k_{\mathrm{B}} T / e_{0}$ ) around one single colloid. Let $n_{\mathrm{s}}$ be the concentration of the monovalent salt. $Z n_{\mathrm{p}}+n_{\mathrm{s}}$ then is the concentration of counterions, $n_{\mathrm{s}}$ that of the co-ions. Within the Poisson-Boltzmann (PB) theory, the charge distribution of the positively and negatively charged microions is given by $\rho_{+}(r)=\left(Z n_{\mathrm{p}}+n_{\mathrm{s}}\right) e^{-\Psi(r)}$ and $\rho_{-}(r)=n_{\mathrm{s}} e^{\Psi(r)}$, respectively. In the JM the requirement of electroneutrality is ensured by assuming that all colloidal particles surrounding the central one contribute equally to a homogeneously distributed background charge density $\rho_{\mathrm{bg}}=Z n_{\mathrm{p}}$. From Poisson's equation, linking $\Psi(r)$ to the total charge density $\rho_{+}-\rho_{-}-\rho_{\mathrm{bg}}$, one then obtains

$$
\Psi^{\prime \prime}(r)+\frac{2}{r} \Psi^{\prime}(r)=-4 \pi l_{\mathrm{B}}\left[\left(Z n_{\mathrm{p}}+n_{\mathrm{s}}\right) e^{-\Psi(r)}-n_{\mathrm{s}} e^{\Psi(r)}-Z n_{\mathrm{p}}\right] \quad(r>a)
$$

with the prime denoting the derivative with respect to the distance $r$ from the central colloid at the origin. $l_{\mathrm{B}}=e_{0}^{2} /\left(\epsilon k_{\mathrm{B}} T\right)$ is the Bjerrum length related to the solvent dielectric constant $\epsilon$. Equation (1), together with the boundary conditions $\Psi^{\prime}(a)=Z l_{\mathrm{B}} / a^{2}$ and $\Psi(\infty)=0$, constitutes a complete boundary value problem (BVP) [15]. If $\Psi$ is small compared to unity, the exponential factors on the right-hand side of the PB equation (1) can be linearized, the $\Psi$ independent terms then cancel due to the imposed electroneutrality and one finds $\nabla^{2} \Psi=\kappa^{2} \Psi$ with $\kappa^{2}=4 \pi l_{\mathrm{B}}\left(Z n_{\mathrm{p}}+2 n_{\mathrm{s}}\right)$, a differential equation which is much easier to solve. For larger colloidal charges $Z$, the condition $\Psi \ll 1$ is fulfilled only at sufficiently large $r$, i.e., if $r>a^{*}$ with $a^{*} \geq a$. In the JM the original particle, characterized by $(Z, a)$, is therefore replaced by another sphere with $\left(Z^{*}, a^{*}\right)$, which now allows linearization of the PB equation. This leads to the BVP

$$
\Psi^{\prime \prime}(r)+\frac{2}{r} \Psi^{\prime}(r)=\kappa^{2} \Psi \quad\left(r>a^{*}\right)
$$

with boundary conditions $\Psi^{\prime}\left(a^{*}\right)=Z^{*} l_{\mathrm{B}} /\left(a^{*}\right)^{2}$ and $\Psi(\infty)=0$. The new radius $a^{*}$ ensures that $\Psi \ll 1$ for all $r>a^{*}$ and where $Z^{*}$ is chosen such that by construction the solutions of the original BVP and the linearized one in eq. (2) are identical at $r>a^{*}$ [16]. The solution of eq. (2) can thus be regarded as the asymptotic far-field solution of eq. (1). Its analytical form is $\Psi(r)=l_{\mathrm{B}} \bar{y} e^{-\kappa r} / r$ with $\bar{y}=Z^{*} e^{\kappa a^{*}} /\left(1+\kappa a^{*}\right)$.

With this asymptotic solution at hand, the effective pair potential $U_{\text {eff }}(r)$ between two colloidal particles at a relative distance $r$ can be obtained by the superposition of the electrostatic potentials $\Psi(r)$ around each of the two colloids and integrating the stress tensor over the mid-plane [3], resulting in $U_{\text {eff }}(r)=k_{\mathrm{B}} T l_{\mathrm{B}} \bar{y}^{2} e^{-\kappa r} / r$. Using this effective pair potential, one can finally determine the structure factor by using one of the well-established approximate methods of liquid-state theory [17]. We here used the i) Rescaled Mean-Spherical Approximation (RMSA) and ii) the Rogers-Young closure relation, and found that the RMSA tends to slightly overestimate the structure by at most $10 \%$ around $q_{\max }$. In view of the smallness of this difference and because the numerical code is more efficient and much faster, we used the RMSA for the systematic computations of $S(q)$ presented in the following. Values of $S\left(q_{\max }\right)$ as a function of $\varphi$ for $Z=300,700,1000$, and 1500 are shown in fig. $2 \mathrm{~b}$. The input parameters correspond to the experimental conditions in fig. 1 , with a very small amount $\left(10^{-7} \mathrm{M}\right)$ of monovalent added salt to represent the residual concentrations of $\mathrm{H}^{+}$and $\mathrm{OH}^{-}$ after treatment with ion-exchange resin. The only unknown parameter left then is the bare colloidal charge $Z$; that is why we have varied this quantity. Based on PB cell model calculations including charge regulation [18], we assume in the following that the particle charge is independent of $\varphi$ due to the strongly acidic sulfate groups at the particle surface.

To discuss our results, we first note that the $\varphi$ regime where $S\left(q_{\max }\right)$ is dominated by the counterions can be recognized from fig. $2 \mathrm{~b}$ to lie above $\varphi=10^{-4}$ roughly; below this 
value all curves converge because salt-ion screening is dominating. Comparing $S\left(q_{\max }\right)$ vs. $\varphi$ curves calculated on the basis of density-dependent and density-independent pair potentials, Beresford-Smith et al. pointed out that it is due to the density dependence of $U_{\text {eff }}(r)$ that charge-stabilized colloidal suspension can show a liquid-like structure over up to four orders of magnitude in $\varphi$, quite in contrast to a system with density-independent potentials which starts crystallizing within an order-of-magnitude increase in $\varphi$. A liquid-like structure over many orders of magnitude in $\varphi$ is indeed observed in our experiment. According to the JM, the density dependence of the pair potential furthermore arises through the quantities $\kappa$ and $\bar{y}$ specifying the Yukawa potential. The screening constant $\kappa=4 \pi l_{\mathrm{B}}\left(Z n_{\mathrm{p}}+2 n_{\mathrm{s}}\right)^{1 / 2}$ decreases monotonically with decreasing $Z \varphi$. This leads to an increase of the range of $U_{\text {eff }}(r)$ with decreasing $\varphi$ and/or $Z$, and hence to the seemingly paradoxical result of enhanced structure despite decreasing $Z$, see fig. $2 \mathrm{~b}$. On the other hand, $\bar{y}$ as a function of $Z \varphi$ shows a nonmonotonic behavior with a well-resolved minimum [3]. The combined effect of both quantities in $U_{\text {eff }}(r)$ explains the reappearance of structure with decreasing $\varphi$, that is, the formation of a shallow minimum in the $S\left(q_{\max }\right)$ curves of fig. $2 \mathrm{~b}$ at high $Z$. This central prediction of the $\mathrm{JM}$ is qualitatively confirmed by our experimental data.

Despite this qualitative agreement, the JM severely underestimates the amplitudes of the first peak in $S(q)$. The largest values for $S\left(q_{\max }\right)$ were obtained for $Z=300$; smaller and higher values of $Z$ resulted again in less structure. Therefore, the best agreement possible between theory and experiment is that between the $Z=300$ curve in fig. $2 \mathrm{~b}$ and the experimental data in fig. 2a. That $S\left(q_{\max }\right)$ is invariably too small can be traced back to the screening constant $\kappa$ which in the JM is generally overestimated. This is due to the implicit assumption that screening in the $\mathrm{JM}$ (i.e. the screening constant $\kappa$ ) is determined by all the $Z n_{\mathrm{p}} V$ counterions. A more realistic procedure to derive $\varphi$-dependent effective colloid potentials should account for the strong binding of a fraction of the counterions to the macroion surface leading to a smaller amount of free counterions, a reduction of the screening and, thus, increased structure. The concept of charge renormalization within the PB cell model [19] is a common way to take this effect into account, essentially by using effective colloidal charges $\tilde{Z}$ in $U_{\text {eff }}(r)$. No theory, however, currently exists combining the ideas of the JM with those of charge renormalization. We have also checked the predictions of the PB cell model alone. We calculated $\tilde{Z}$ following [19]; then the density-dependent effective pair potentials and finally $S\left(q_{\max }\right)$ were computed in the RMSA (data not shown here). We found that $S\left(q_{\max }\right)$ is generally much higher when compared to the JM results, but does now monotonically increase with $\varphi$, with just a soft shoulder but no minimum at all. Consequently, the PB cell model fails to reproduce the observed reappearance of structure.

We may conclude from this discussion that although the experimentally observed reappearance of structure can be understood qualitatively within the jellium model, this model seems to be inadequate to give a satisfactory quantitative agreement between theory and experiment. One reason for the observed discrepancy might be that at such a low ionic strength one of our basic assumptions breaks down, namely that the total free energy of the suspension can be approximated by a sum of effective Yukawa-like pair potentials. The present work is not the first experimental study on highly deionized colloidal suspensions where many-body effects seem to play a crucial role. We just mention the recent work of Brunner et al. [20] providing a direct experimental observation of many-body forces in 2D colloidal suspensions, or the study of Yamanaka et al. [21] who have observed a peculiar re-entrant solid-liquid phase transition in a deionized suspension of silica particles which is not explainable in terms of pairwise effective Yukawa potentials. Remarkably enough, their results have been found in a parameter regime that is not much different from ours. 
We thank J. KoHLBRECHER for help with the SANS experiments, and A. BANCHIO and G. NÄGELE for help with the RY code.

\section{REFERENCES}

[1] Verwey E. J. W. and Overbeek J. T. G., Theory of the Stability of Lyophobic Colloids (Elsevier, New York) 1948.

[2] Belloni L., Colloids Surf. A, 140 (1998) 227.

[3] Beresford-Smith B., Chan D. Y. C. and Mitchell D. J., J. Colloid Interface Sci., 216 (1985) 9691.

[4] Härtl W. and Versmold H., J. Chem. Phys., 88 (1988) 7157.

[5] Krause R., Nägele G., Karrer D., Schneider J., Klein R. and Weber R., Physica A, $153(1988) 400$.

[6] Quesada-Pérez M., Callejas-Fernández J. and Hidalgo-Álvarez R., Adv. Colloid Interface Sci., 95 (2002) 295.

[7] Bertolini D., Cassettari M. and Salvetti G., J. Chem. Phys., 78 (1983) 365.

[8] Schätzel K., J. Mod. Opt., 38 (1991) 1849.

[9] Aberle L. B., Hülstede P., Wiegand S., Schröer W. and Staude W., Appl. Opt., 37 (1998) 6511.

[10] Urban C. and Schurtenberger P., J. Colloid Interface Sci., 207 (1998) 150.

[11] Overbeck E., Sinn C., Palberg T. and Schätzel K., Colloids Surf. A, 122 (1997) 83.

[12] Urban C., Development of Fiber Optic Based Dynamic Light Scattering for a Characterization of Turbid Suspensions, PhD thesis, ETH Zürich (1999).

[13] D'Aguanno B. and Klein R., Phys. Rev. A, 46 (1992) 7652.

[14] Wagner N. J., Krause R., Rennie A. R., D'Aguanno B. and Goodwin J., J. Chem. Phys., 95 (1991) 494.

[15] The BVP in [3] has a slightly different form, with a $\varphi$-dependent boundary condition (eqs. (4.30) and (4.34) in [3]). To take account of the reduced volume accessible to the microions, we have replaced $\varphi$ in the BVP of [3] by $\varphi /(1-\varphi)$. Using then $Z$ as an abbreviation for $Z_{\text {real }} /(1-\varphi)$ leads to eq. (1). Here, $Z_{\text {real }}$ is the bare charge in [3]. The difference between $Z_{\text {real }}$ and $Z$ is very small in most cases and has been neglected in our calculations.

[16] We define $a^{*}$ to be the radius where $\Psi\left(a^{*}\right)=0.1$. This choice of $a^{*}$ ensures that $\Psi \ll 1$ for all $r>a^{*}$. Taking $\Psi^{\prime}$ at $a^{*}$ with $\Psi(r)$ solving eq. (1), one can calculate a $Z^{*} l_{\mathrm{B}}=\left.\Psi^{\prime}\right|_{r=a^{*}}\left(a^{*}\right)^{2}$ and then use $\left(Z^{*}, a^{*}\right)$ to set up the linearized BVP in eq. (2). By construction, the solution to this BVP is identical to that of eq. (1) at $r>a^{*}$.

[17] Klein R. and D'Aguanno B., in Light Scattering. Principles and Development, edited by Brown W. (Clarendon Press, Oxford) 1996, p. 30.

[18] Gisler T., Schulz S. F., Borkovec M., Sticher H., Schurtenberger P., D'Aguanno B. and Klein R., J. Chem. Phys., 101 (1994) 9924.

[19] Alexander S., Chaikin P. M., Grant P., Morales G. J., Pincus P. and Hone D., J. Chem. Phys., 80 (1984) 5776.

[20] Brunner M., Bechinger C., Strepp W., Lobaskin V. and von Grünberg H. H., Europhys. Lett., 58 (2002) 926.

[21] Yamanaka J., Yoshida H., Koga T., Ise N. and Hashimoto T., Phys. Rev. Lett., 80 (1998) 5806 . 